\title{
Do known risk factors explain the higher coronary heart disease mortality in South Asian compared with European men? Prospective follow-up of the Southall and Brent studies, UK
}

\author{
N. G. Forouhi • N. Sattar • T. Tillin • P. M. McKeigue • \\ N. Chaturvedi \\ Received: 10 May 2006 / Accepted: 30 June 2006 / Published online: 14 September 2006 \\ (C) Springer-Verlag 2006
}

\begin{abstract}
Aims/hypothesis We examined prospectively whether mea-
Abstract
Aims/hypothesis We examined prospectively whether measured risk factors can explain the higher CHD mortality in South Asians compared with Europeans.

Materials and methods Conventional CHD risk factors and those associated with insulin resistance were measured in 1,787 European and 1,420 South Asian men aged 40 to 69 years at baseline in the population-based Southall and Brent studies (London) between 1988 and 1990. Participants were followed up for mortality.

Results By February 2006, there were 202 CHD deaths (108 Asian, 94 European). South Asian men had double the CHD mortality of European men in Cox regression analyses adjusted for age, smoking, and cholesterol (hazard ratio [HR] 2.14, 95\% CI 1.56-2.94, $p<0.001)$. Nearly half of all South Asian CHD deaths versus 13\% of deaths among Europeans were among persons with diabetes. Asian men had greater CHD mortality than Europeans,
\end{abstract}

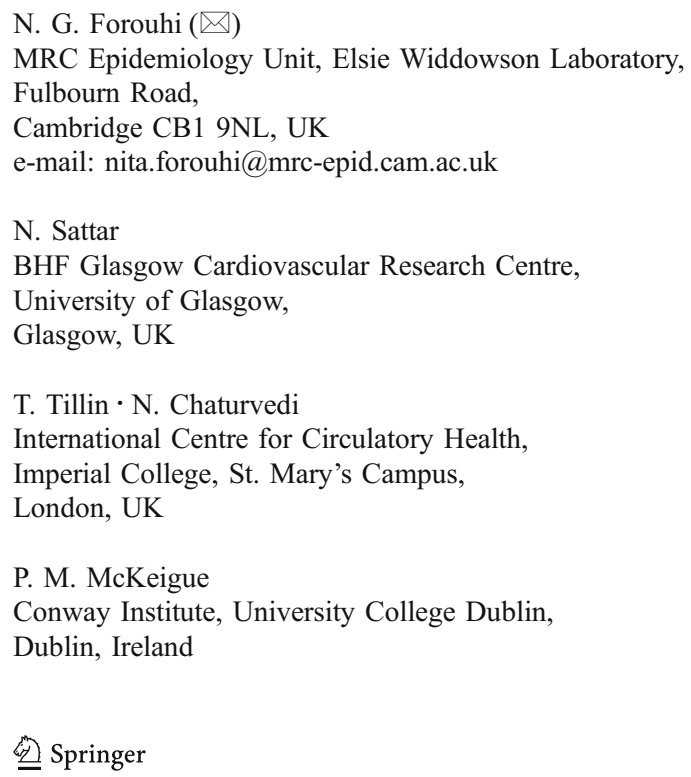

both in the with- and the without-diabetes categories at baseline. CHD mortality remained significantly higher in South Asian men in multivariable models that adjusted for conventional risk factors and diabetes and/or impaired glucose regulation, features of insulin resistance, or the metabolic syndrome (HR 1.6-1.9). Accounting for comorbidity and socio-economic status did not materially alter the findings.

Conclusions/interpretation These data confirm that South Asian men have significantly higher CHD mortality than their European counterparts, while indicating that neither conventional risk factors, nor insulin resistance parameters or metabolic syndrome criteria as currently defined can account for this excess risk. The contribution of unmeasured factors to the elevated vascular risk in South Asians should be addressed in future studies.

Keywords Coronary heart disease $\cdot$ Ethnicity $\cdot$ Mortality . South Asian

\begin{tabular}{ll}
\multicolumn{2}{l}{ Abbreviations } \\
HOMA & homeostasis model assessment \\
HOMA-IR & $\begin{array}{l}\text { homeostasis model assessment insulin } \\
\text { resistance index }\end{array}$ \\
HR & hazard ratio \\
ICD & International Classification of Diseases \\
IDF & International Diabetes Federation
\end{tabular}

\section{Introduction}

People of South Asian descent (Indian, Pakistani or Bangladeshi origin) who migrate to the Western world have one of the highest rates of CHD in the world, being 50 
to $200 \%$ higher than that of comparator European populations [1-3]. Within each ethnic group conventional risk factors such as smoking, hypertension and total cholesterol are of immense importance in predicting absolute CHD risk, e.g. the risk of CHD in current smokers compared with never-smokers is elevated both in Europeans and South Asians. However, important as they are within the South Asian group, conventional risk factors do not account for the greater prevalence of CHD in South Asians compared to Europeans; in other words: they do not account for between-ethnic-group differences in CHD risk [3]. Results of cross-sectional studies [3-6] and case-control studies $[7,8]$ have supported the hypothesis that insulin resistance may underlie the high rates of CHD in South Asians, but it has also been suggested that alternative explanations need to be sought $[9,10]$.

From analysis of national routine data, it has been reported that $\mathrm{CHD}$ mortality is $50 \%$ higher in people born in the Indian sub-continent than in people born in England [2]. However, prospective data examining CHD mortality in South Asians compared with other ethnic groups are sparse. The only relevant data in this respect confirmed the high rates of CHD mortality in South Asians in Trinidad [11] and Fiji [12], but did not study associations with the full range of conventional risk factors or features of the metabolic syndrome. Our objectives, therefore, were: (1) to prospectively measure the CHD mortality rate in European and South Asian men in the United Kingdom in the population-based Southall and Brent studies [4, 13]; and (2) to test whether any of the measured risk factors at baseline could account for the different CHD mortality between the ethnic groups.

\section{Subjects and methods}

\section{Study design and participants}

We used data on 3,207 European and South Asian men from the Southall [3, 4] and Brent [13] population-based studies, which were conducted to identical protocols between 1988 and 1991 in West London and followed since then for deaths and causes of death. Both studies have previously been described in detail [3, 4, 13] and were approved by local research ethics committees, with written informed consent provided by all participants. All participants were aged 40 to 69 years at baseline and the total available sample in the pooled Southall and Brent studies included 3,711 men, from which we excluded 456 African/ Caribbean men and 48 men of other ethnic origin, leaving a final sample of 1,787 European men and 1,420 South Asian men for this study. Main recruitment was from age-, ethnicity- and sex-stratified random samples from general practice lists in the London boroughs of Southall and Brent (77\% of the sample). An additional sample (23\%) was assembled from four West London factories, chosen on the basis of the ethnic mix of their workforce. Men with cancer, renal failure, severe disability or severe psychiatric disturbance were excluded. Ethnicity was identified on the basis of name, country of birth and appearance, supplemented by direct enquiry in cases of doubt.

\section{Baseline measurements}

Following an overnight fast, measurements to standard protocol (described elsewhere in detail [4]) included: anthropometry (height, weight, waist and hip girth - all measured in the standing position), blood pressure, resting electrocardiogram and blood samples for fasting glucose, insulin and lipids (plasma total and HDL-cholesterol, plasma triglycerides). Among participants with no history of diabetes mellitus, a 75-g OGTT was performed with glucose and insulin measurements at $2 \mathrm{~h}$. A self-administered questionnaire included items on smoking, medical history, socio-economic status (years of education and occupation), and physical activity (during work, leisure time, travel time and sports). Laboratory measurements for the two studies were performed at the same hospital chemical pathology laboratory.

Smoking was categorised as never smoker, ex-smoker and current smoker. Impaired glucose tolerance, impaired fasting glucose and diabetes were defined according to WHO 1999 criteria [14]. Social class was assigned according to the Registrar General's classification in use at the time of the baseline survey and categories of manual and non-manual social class derived. Duration of education was classified as $<11$ or $\geq 11$ years. For physical activity we derived an overall exercise score from activity during work, leisure, sports and travel time.

Metabolic syndrome was defined by the recent ethnicityspecific consensus of the International Diabetes Federation (IDF) [15] as follows: waist circumference $\geq 94 \mathrm{~cm}$ European men or $\geq 90 \mathrm{~cm}$ South Asian men, plus any two of the following four factors: (1) fasting glucose $\geq 5.6 \mathrm{mmol} / 1$ (100 mg/dl) or previously diagnosed diabetes; (2) triglycerides $\geq 1.7 \mathrm{mmol} / 1 \quad(150 \mathrm{mg} / \mathrm{dl})$ or specific treatment for this lipid abnormality; (3) low HDL-cholesterol $<1.03 \mathrm{mmol} / \mathrm{l}$ in men $(40 \mathrm{mg} / \mathrm{dl})$ or specific treatment for this lipid abnormality; or (4) high blood pressure $(\geq 130 / \geq 85 \mathrm{mmHg}$ systolic or diastolic blood pressure, or antihypertensive medication use).

\section{Mortality follow-up}

All participants were flagged for death notification from the Office for National Statistics, with cause of death coded 
according to the International Classification of Diseases (ICD), ninth or tenth revisions. We defined CHD mortality as death with mention anywhere on the death certificate of ICD-9 codes 410.0 to 414.9 or ICD-10 codes I200 to I250. Individual follow-up time was taken into account by calculation of person-years at risk up to 14 February 2006 or date of death or date of recorded loss to follow-up among survivors. The follow up rate was $99.4 \%$.

\section{Statistical analyses}

Glucose, insulin, triglycerides and HDL-cholesterol were $\log$ normalised. Our a priori analysis plan was as follows. First, we examined baseline characteristics in each ethnic group to set out the distribution of risk factors by ethnic group. Tests of significance for proportions were by the chisquared statistic, and of difference in mean values by the $t$ test. Second, we examined the univariate association of the potential risk factors (independent variables) with $\mathrm{CHD}$ mortality (dependent variable) within each ethnic group for age, and for other variables adjusted for age, using Cox proportional hazards regression analyses. The third step was to test for an ethnic difference in CHD mortality. Survival time analysis was used to calculate incidence rate per 1,000 person-years in each ethnic group. Multivariable Cox regression models were developed, which tested for an ethnic difference (South Asian coded 1, European coded 0) in CHD mortality in analyses adjusted for age, conventional CHD risk factors (age, smoking, blood pressure, total cholesterol, HDL-cholesterol and additionally diabetes) and for novel risk factors to include features associated with the insulin resistance syndrome. For the latter we used three approaches, the first with continuously distributed variables (glucose, insulin, triglyceride, HDL-cholesterol, and waist circumference), a second using the categorical variable derived as a single composite measure (metabolic syndrome as defined by the IDF), and a third using insulin resistance as approximated by the homeostasis model
Table 1 Baseline characteristics of European and South Asian men in the Southall and Brent studies

Unless otherwise stated, values are mean $(\mathrm{SD})$ or geometric mean $(95 \% \mathrm{CI})$.

IDF International Diabetes Federation

\begin{tabular}{|c|c|c|c|}
\hline Variable & European & South Asian & $p$ value \\
\hline$N$ & 1,787 & 1,420 & \\
\hline Age (years) & $52.9(7.1)$ & $51.2(7.0)$ & $<0.001$ \\
\hline Dead, $n(\%)$ & $329(18.4)$ & $207(14.6)$ & 0.004 \\
\hline CHD death, $n(\%)$ & $94(5.3)$ & $108(7.6)$ & 0.007 \\
\hline \multicolumn{4}{|l|}{ Smoker, $n(\%)$} \\
\hline Never & $514(28.8)$ & $1,047(73.9)$ & \\
\hline Ex & 707 (39.6) & $152(10.7)$ & \\
\hline Current & $563(31.6)$ & $217(15.3)$ & $<0.001$ \\
\hline Manual social class $n(\%)$ & $1,122(63.0)$ & $1,086(76.9)$ & $<0.001$ \\
\hline Education $\geq 11$ years, $n(\%)$ & $699(39.4)$ & $953(71.2)$ & $<0.001$ \\
\hline BMI $\left(\mathrm{kg} / \mathrm{m}^{2}\right)$ & $26.2(3.8)$ & $25.9(3.3)$ & 0.007 \\
\hline Waist circumference $(\mathrm{cm})$ & $92.0(11.0)$ & $93.3(9.7)$ & $<0.001$ \\
\hline WHR & $0.94(0.07)$ & $0.98(0.06)$ & $<0.001$ \\
\hline Systolic BP (mmHg) & $123.0(16.7)$ & $125.4(17.5)$ & $<0.001$ \\
\hline Diastolic BP (mmHg) & $77.2(10.8)$ & $80.9(10.6)$ & $<0.001$ \\
\hline Total cholesterol (mmol/l) & $5.9(1.1)$ & $5.8(1.1)$ & $<0.001$ \\
\hline HDL-cholesterol (mmol/1) & $1.2(1.2-1.3)$ & $1.1(1.1-1.2)$ & $<0.001$ \\
\hline Fasting triglyceride $(\mathrm{mmol} / \mathrm{l})$ & $1.4(1.4-1.5)$ & $1.7(1.6-1.8)$ & $<0.001$ \\
\hline Fasting glucose $(\mathrm{mmol} / \mathrm{l})$ & $5.6(5.5-5.6)$ & $6.0(5.9-6.1)$ & $<0.001$ \\
\hline 2-h glucose $(\mathrm{mmol} / \mathrm{l})$ & $5.0(4.9-5.1)$ & $5.9(5.7-6.0)$ & $<0.001$ \\
\hline Fasting insulin (pmol/1) & $51.1(49.6-52.6)$ & $75.0(72.5-77.6)$ & $<0.001$ \\
\hline 2-h insulin (pmol/1) & $139.3(133.9-144.8)$ & $286.8(273.8-300.4)$ & $<0.001$ \\
\hline HOMA-IR & $1.8(1.8-1.9)$ & $2.9(2.8-3.0)$ & $<0.001$ \\
\hline Diabetes, new or known, $n(\%)$ & $127(7.2)$ & $311(22.1)$ & $<0.001$ \\
\hline Diabetes or IFG or IGT, $n(\%)$ & $177(19.6)$ & $382(35.4)$ & $<0.001$ \\
\hline Metabolic syndrome (IDF), $n(\%)$ & $404(23.1)$ & $612(44.6)$ & $<0.001$ \\
\hline \multicolumn{4}{|c|}{ Component of metabolic syndrome (IDF), $n(\%)$} \\
\hline High waist circumference & $681(38.2)$ & $890(62.7)$ & $<0.001$ \\
\hline Glucose Impairment & $692(38.9)$ & $724(51.2)$ & $<0.001$ \\
\hline Low HDL & $428(24.4)$ & $507(36.8)$ & $<0.001$ \\
\hline High triglyceride & $629(35.3)$ & $682(48.2)$ & $<0.001$ \\
\hline Hypertensive & $712(39.9)$ & $738(52.0)$ & $<0.001$ \\
\hline
\end{tabular}


assessment (HOMA) insulin resistance index (HOMA-IR) [16]. The final step was to examine ethnic difference in CHD mortality in persons with and without diabetes. For continuous variables the hazard ratios (HRs) reflect risk associated with a unit change in the explanatory variable.

The effect of other potential confounders was also tested in models that adjusted for socioeconomic factors and physical activity. We tested for statistical interaction between ethnic groups and each of the risk factors in relation to $\mathrm{CHD}$ mortality. All analyses were performed using STATA (version 8; StataCorp, College Station, TX, USA). We tested for the proportional hazards assumption in both observed and analytical ways (log-log plot and 'stphtest' command with Schoenfeld residuals in STATA respectively), and found the assumption to be valid.

\section{Results}

Baseline characteristics

At baseline, South Asians were slightly younger, and had fewer smokers, lower BMI and lower total cholesterol than European men. However, features associated with insulin resistance were more adversely distributed among South Asians (Table 1).

\section{CHD mortality}

The median follow-up time was 16.2 years (range: $0.02-$ 17.68 years). Of the 3,207 total, 20 men were lost to follow-up, giving an excellent follow-up rate of $99.4 \%$. There were 536 deaths, of which 202 were CHD deaths (94 European, 108 South Asian).

\section{CHD mortality within each ethnic group}

Univariate associations of measured risk factors with $\mathrm{CHD}$ mortality within each of the groups are shown in Table 2. Increasing age, and in age-adjusted analyses, smoking, hypertension, fasting glucose and presence of diabetes/ impaired glucose regulation (IFG or IGT) were significantly associated with CHD mortality in men of both ethnic groups (Table 2). Among European men, total cholesterol, fasting triglyceride, 2-h insulin and the IDF definition of metabolic syndrome were also significantly associated with CHD mortality. Among South Asian men, significant risk factors for CHD mortality were HOMA-IR and diabetes. Greater duration of education protected against CHD mortality in South Asians.

\section{Ethnic difference in CHD mortality}

The crude CHD mortality incidence rate per 1000 personyears was 3.5 [95\% CI 2.9-4.3] in Europeans and 4.9
Table 2 Age-adjusted univariate Cox regression analysis of associations of measured risk factors with CHD mortality in European and South Asian men: Southall and Brent Prospective Study

IDF International Diabetes Federation

${ }^{\mathrm{a}} \log$ (natural) transformed

\begin{tabular}{|c|c|c|c|c|}
\hline Risk factor & European men & & South Asian men & \\
\hline \multirow[t]{2}{*}{ Number total:deaths } & $1,787: 94$ & & $1,420: 108$ & \\
\hline & Hazard ratio $(95 \% \mathrm{CI})$ & $p$ value & Hazard ratio $(95 \% \mathrm{CI})$ & $p$ value \\
\hline Age (years) (unadjusted) & $1.14(1.10-1.17)$ & $<0.001$ & $1.10(1.07-1.13)$ & $<0.001$ \\
\hline \multicolumn{5}{|l|}{ Smoking } \\
\hline $\mathrm{Ex}$ & $1.46(0.81-2.64)$ & 0.21 & $1.48(0.85-2.58)$ & 0.17 \\
\hline Current & $2.68(1.51-4.77)$ & 0.001 & $1.86(1.15-3.00)$ & 0.011 \\
\hline Manual occupation & $0.94(0.61-1.43)$ & 0.76 & $1.62(0.97-2.72)$ & 0.068 \\
\hline Education $\geq 11$ years & $0.94(0.59-1.47)$ & 0.77 & $0.53(0.36-0.79)$ & 0.002 \\
\hline BMI $\left(\mathrm{kg} / \mathrm{m}^{2}\right)$ & $1.04(0.99-1.10)$ & 0.097 & $0.99(0.94-1.05)$ & 0.80 \\
\hline Waist circumference $(\mathrm{cm})$ & $1.01(1.00-1.03)$ & 0.14 & $1.00(0.98-1.02)$ & 0.75 \\
\hline $\begin{array}{l}\text { Hypertension }(>140 / 90 \text { or } \\
\text { treatment) }\end{array}$ & $2.49(1.65-3.76)$ & $<0.001$ & $2.35(1.59-3.47)$ & $<0.001$ \\
\hline Total cholesterol (mmol/1) & $1.20(1.01-1.42)$ & 0.036 & $1.14(0.96-1.36)$ & 0.13 \\
\hline HDL-cholesterol $^{\mathrm{a}}(\mathrm{mmol} / \mathrm{l})$ & $0.52(0.24-1.15)$ & 0.11 & $0.50(0.24-1.05)$ & 0.07 \\
\hline Fasting triglyceride ${ }^{\mathrm{a}}(\mathrm{mmol} / \mathrm{l})$ & $1.66(1.15-2.41)$ & 0.007 & $1.30(0.94-1.80)$ & 0.12 \\
\hline Fasting glucose $\mathrm{e}^{\mathrm{a}}(\mathrm{mmol} / \mathrm{l})$ & $2.18(1.03-4.61)$ & 0.042 & $3.28(1.98-5.43)$ & $<0.001$ \\
\hline 2-h glucose ${ }^{\mathrm{a}}(\mathrm{mmol} / \mathrm{l})$ & $1.01(0.56-1.84)$ & 0.96 & $2.13(1.35-3.34)$ & 0.001 \\
\hline Fasting insulin ${ }^{\mathrm{a}}(\mathrm{pmol} / \mathrm{l})$ & $1.24(0.90-1.71)$ & 0.19 & $1.31(0.99-1.72)$ & 0.058 \\
\hline 2-h insulin ${ }^{\mathrm{a}}(\mathrm{pmol} / \mathrm{l})$ & $1.40(1.07-1.83)$ & 0.015 & $1.05(0.80-1.38)$ & 0.73 \\
\hline HOMA insulin resistance ${ }^{a}$ & $1.23(0.94-1.62)$ & 0.13 & $1.42(1.14-1.77)$ & 0.002 \\
\hline Diabetes (new or known) & $1.46(0.79-2.68)$ & 0.23 & $2.78(1.89-4.09)$ & $<0.001$ \\
\hline Diabetes or IGT & $1.81(1.09-3.01)$ & 0.021 & $2.74(1.87-4.03)$ & $<0.001$ \\
\hline Diabetes or IFG or IGT & $1.57(1.01-2.43)$ & 0.044 & $2.23(1.52-3.28)$ & $<0.001$ \\
\hline Metabolic syndrome (IDF) & $1.61(1.04-2.48)$ & 0.031 & $1.25(0.85-1.83)$ & 0.26 \\
\hline
\end{tabular}


[4.1-6.0] in South Asians. The greater CHD mortality rate in South Asian men is illustrated in the Kaplan-Meier survival estimates (Fig. 1).

In age-adjusted analyses, CHD mortality was 60\% greater in South Asians than in Europeans (Table 3). In multivariable analyses, additional adjustment for smoking and total cholesterol concentration led to a doubling of the CHD mortality rate in South Asians compared with Europeans (HR 2.14, 95\% CI 1.56-2.94, $p<0.001$ ). Table 3 shows that the CHD mortality rate in South Asian men remained significantly elevated in all the models we tested, with or without adjustment for socioeconomic status. Thus South Asian men had significantly higher CHD mortality after adjustment for conventional risk factors (including age, cholesterol, smoking, HDL-cholesterol, blood pressure and diabetes), and also after adjusting for 'novel' risk factors (insulin resistance measured by HOMA, or features associated with insulin resistance, or component or composite definitions of metabolic syndrome). There was no significant interaction between ethnicity and any of the risk factors including diabetes $(p=0.11)$, or metabolic syndrome $(p=0.25)$. However, it should be borne in mind that our study was not specifically powered to test for interactions.

Results were of similar magnitude and direction when additionally controlling for the effect of physical activity levels or prevalent CHD at baseline. We also accounted for the potential effect of co-morbidity by excluding from analysis men who died in the first 5 years of follow-up, and by adjusting for self-reported health at baseline. In both instances, the CHD mortality rate remained 50 to $60 \%$ higher in Asian men in analyses adjusted for age, total cholesterol, smoking, and diabetes/impaired glucose regulation. Notably, inclusion of the calculated concentration of LDL-cholesterol in place of total cholesterol did not alter the findings.

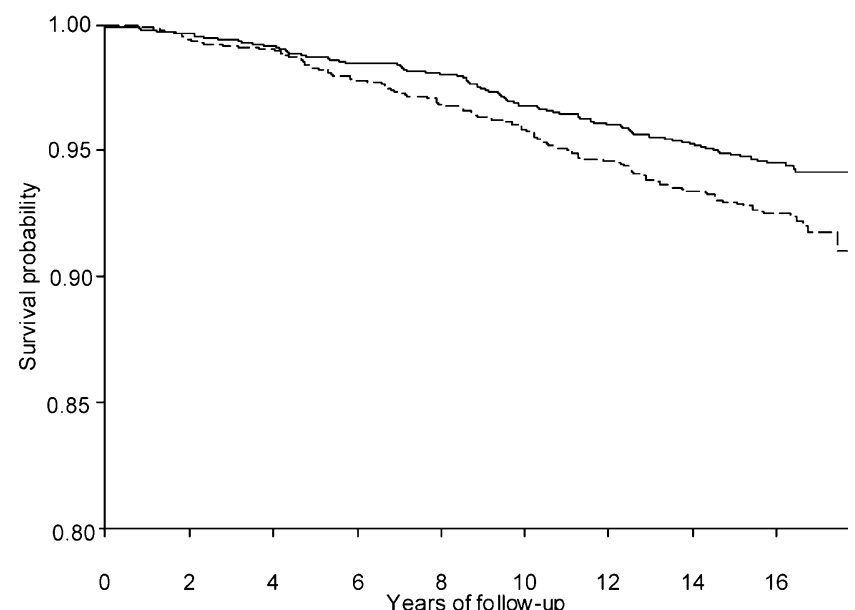

Fig. 1 Kaplan-Meier survival curves of the ethnic difference in CHD mortality among European men (continuous line) and South Asian men (broken line) in the Southall and Brent studies, London
Although the magnitude of the ethnic difference in CHD mortality was greater among those with diabetes than those without, in multivariable analyses there was a trend towards greater CHD mortality in South Asians among all glycaemic groups (Table 4).

\section{Discussion}

This is the first prospective comparative mortality study in a general population-based sample of South Asian migrants to the UK. Our finding of a $60 \%$ greater rate of CHD death in South Asian than in European men is consistent with routine mortality data [2]. We take these latter data further by showing that statistical adjustment for the key conventional CHD risk factors, smoking and total cholesterol, both factors favourably distributed in South Asian migrants, increased the relative risk of death in South Asians versus Europeans from 1.6- to 2.0-fold. Adverse risk factors in South Asians include insulin resistance, dyslipidaemia and hyperglycaemia. Surprisingly, statistical adjustment for the adverse distribution of these variables in South Asians did not account for the elevated risk of CHD in South Asians, which remained 60 to $90 \%$ higher in South Asians after adjustment for these factors.

However, there was a marked ethnic difference in the impact of diabetes on mortality risk. Nearly half of all deaths in South Asians occurred in individuals with diabetes at baseline; this figure was only $13 \%$ in Europeans. In the South Asian group, diabetes increased the mortality risk nearly threefold compared with South Asians without diabetes at baseline, whereas in Europeans, the excess mortality associated with diabetes was only 1.5 -fold. The ethnic difference in CHD mortality persisted in subjects with diabetes in our study, with a doubling of CHD mortality in South Asians. This finding is at variance with that reported from the UK Prospective Diabetes Study (UKPDS 32), where there was no significant ethnic difference in fatal and non-fatal myocardial infarction among Europeans $(n=4,101)$ and South Asians $(n=490)$ with newly diagnosed diabetes (HR $1.2,95 \%$ CI $0.9-1.7$ in analyses adjusted for known risk factors) [17]. Possible reasons for this difference could be: (1) the lower age of the UKPDS cohort (25 to 65 years); (2) the setting of a clinical trial rather than a study with 'free-living' participants; (3) the fact that UKPDS subjects were newly diagnosed with diabetes; and (4) the inclusion of both fatal and non-fatal events in UKPDS. However, our findings are in accord with our previous reports based on death certificate data, where we found a twofold to fourfold higher CHD mortality rate in South Asian than in European diabetic patients $[18,19]$. Our current findings are novel, as we report for the first 
Table 3 CHD mortality in South Asian versus European men: Cox regression analyses for models with and without adjustment for socioeconomic status; the Southall and Brent Studies

\begin{tabular}{|c|c|c|c|}
\hline CHD mortality in S. Asian (108 events) versus European (94 events) men, adjusted for: & Hazard ratio & $95 \% \mathrm{CI}$ & $p$ value \\
\hline \multicolumn{4}{|l|}{ Age } \\
\hline Model 1 & 1.64 & $1.24-2.16$ & $<0.001$ \\
\hline Model 2 & 1.82 & $1.34-2.47$ & $<0.001$ \\
\hline \multicolumn{4}{|l|}{ Age, smoking, total cholesterol } \\
\hline Model 1 & 2.14 & $1.56-2.94$ & $<0.001$ \\
\hline Model 2 & 2.29 & $1.63-3.23$ & $<0.001$ \\
\hline \multicolumn{4}{|l|}{ Age, smoking, total cholesterol, HDL-cholesterol, blood pressure ${ }^{\mathrm{a}}$, diabetes ${ }^{\mathrm{b}}$} \\
\hline Model 1 & 1.68 & $1.21-2.34$ & 0.002 \\
\hline Model 2 & 1.76 & $1.23-2.51$ & 0.002 \\
\hline \multicolumn{4}{|l|}{ Age, smoking, total cholesterol, HDL-cholesterol, blood pressure ${ }^{\mathrm{a}}$, HOMA-IR ${ }^{\mathrm{c}}$} \\
\hline Model 1 & 1.81 & $1.30-2.53$ & 0.001 \\
\hline Model 2 & 1.90 & $1.33-2.74$ & $<0.001$ \\
\hline \multicolumn{4}{|l|}{ Age, smoking, total cholesterol + component features of insulin resistance ${ }^{\mathrm{d}}$} \\
\hline Model 1 & 1.75 & $1.19-2.58$ & 0.005 \\
\hline Model 2 & 1.82 & $1.20-2.78$ & 0.005 \\
\hline \multicolumn{4}{|l|}{ Age, smoking, total cholesterol + component features of metabolic syndrome ${ }^{\mathrm{e}}$} \\
\hline Model 1 & 1.78 & $1.28-2.46$ & 0.001 \\
\hline Model 2 & 1.88 & $1.32-2.67$ & $<0.001$ \\
\hline \multicolumn{4}{|l|}{$\begin{array}{l}\text { Age, smoking, total cholesterol }+ \text { composite definition of IDF metabolic syndrome (see Subjects } \\
\text { and methods) }\end{array}$} \\
\hline Model 1 & 2.04 & $1.47-2.84$ & $<0.001$ \\
\hline Model 2 & 2.20 & $1.54-3.14$ & $<0.001$ \\
\hline
\end{tabular}

Model 1. Ethnic difference in CHD mortality (S. Asian vs European), adjusted for listed variables. Model 2. Additionally adjusted for socioeconomic status (years of education and occupational social class). Coding of variables: age, cholesterol, HDL-cholesterol, triglyceride, insulin, BMI, waist circumference as continuous variables. Smoking: nonsmoker=0, ex-smoker=1, current smoker=2.

${ }^{\text {a }}$ Tertiles of systolic blood pressure with those on antihypertensive medication in top tertile

${ }^{\mathrm{b}}$ Diabetes new or known (WHO99 definition) $=1$, no diabetes $=0$

${ }^{\mathrm{c}}$ HOMA-IR=homeostasis model assessment insulin resistance index

${ }^{\mathrm{d}}$ Features associated with insulin resistance (continuous variables) included fasting and 2-h glucose and insulin, HDL-cholesterol, fasting triglyceride and waist circumference

time the CHD mortality experience by ethnic group among a general population of Europeans and South Asians that included individuals with and without diabetes.

One key question, given the greater prevalence of diabetes, and its marked association with CHD death in South Asians in our study, is: why did the presence of diabetes fail to account for the higher CHD mortality experience of South Asians? One possibility is that standard methods of statistical adjustment to account for or explain group differences in disease risk have limitations, as several assumptions are made about equivalence of effect between groups and linearity of impact across the range of a given risk factor. Our demonstration of a differential impact of diabetes on CHD death by ethnicity illustrates this problem. Another possibility is that we are unable to capture the full effect of diabetes because of our inability to account for disease duration. It is known that South Asians may develop diabetes more than 10 years earlier on average than Europeans [20]. Conceivably, much of the ethnic difference between those with and without diabetes is attributable to the different duration of diabetes in the two groups, and this should be the subject of future study. In an earlier study of diabetic individuals aged 30 to 64 years, we reported a doubling of CHD mortality risk in 730 diabetic South Asians compared with 304 diabetic Europeans in analyses adjusted for age, sex and diabetes duration [19]. However, we cannot rule out the possibility that other unmeasured risk factors, whether associated with insulin resistance or not, could further account for the excess risk of CHD in South Asians. Indeed in our study, we report higher CHD mortality in South Asians than in Europeans within the whole group, as well as among persons with and without diabetes, the magnitude of association being higher in people with diabetes.

In our study, the significantly higher CHD mortality in South Asian men persisted after adjusting for conventional risk factors as well as for factors associated with insulin resistance or with component or composite definitions of the metabolic syndrome. Factors known to be associated with insulin resistance can be better assessed than by the limited methods available for our study. For example, a single fasting triglyceride measurement is not representative 
Table 4 CHD mortality in South Asian versus European men: Cox regression analyses for the whole group, those without diabetes and those with diabetes at baseline; the Southall and Brent Studies

${ }^{\mathrm{a}}$ Missing data for assignment of diabetes status, giving different totals from Table 1. HOMA-IR, homeostasis model assessment index of insulin resistance; IDF, International Diabetes Federation

\begin{tabular}{|c|c|c|c|}
\hline & All & No diabetes & Diabetes \\
\hline$n$ : Total/European/South Asian & $3,182 / 1,774 / 1,408^{\mathrm{a}}$ & $2,744 / 1,647 / 1,097$ & $438 / 127 / 311$ \\
\hline $\begin{array}{l}n: \text { CHD deaths, Total/European/ } \\
\text { South Asian }\end{array}$ & 202/94/108 & $139 / 82 / 57$ & $63 / 12 / 51$ \\
\hline $\begin{array}{l}\text { Ethnic difference in CHD mortality } \\
\text { adjusted for listed variables: }\end{array}$ & Hazard ratio $(95 \% \mathrm{CI})$ & & \\
\hline Age & $1.64(1.24-2.16)$ & $1.24(0.88-1.75)$ & $1.95(1.03-3.67)$ \\
\hline$p$ value & $<0.001$ & 0.21 & 0.040 \\
\hline Age, smoking, total cholesterol & $2.14(1.56-2.94)$ & $1.66(1.13-2.44)$ & $2.29(1.14-4.58)$ \\
\hline$p$ value & $<0.001$ & 0.010 & 0.020 \\
\hline $\begin{array}{l}\text { Age, smoking, total cholesterol, } \\
\text { HDL-cholesterol, blood pressure }\end{array}$ & $1.92(1.39-2.66)$ & $1.50(1.02-2.22)$ & $2.34(1.14-4.78)$ \\
\hline$p$ value & $<0.001$ & 0.041 & 0.020 \\
\hline $\begin{array}{l}\text { Age, smoking, total cholesterol, } \\
\text { HDL-cholesterol, blood pressure, } \\
\text { HOMA-IR }\end{array}$ & $1.81(1.30-2.53)$ & $1.58(1.06-2.36)$ & $2.46(1.16-5.20)$ \\
\hline$p$ value & 0.001 & 0.025 & 0.018 \\
\hline $\begin{array}{l}\text { Age, smoking, total cholesterol + } \\
\text { component features of metabolic } \\
\text { syndrome (continuous) }\end{array}$ & $1.79(1.29-2.48)$ & $1.48(1.00-2.20)$ & $2.33(1.14-4.78)$ \\
\hline$p$ value & 0.001 & 0.049 & 0.021 \\
\hline $\begin{array}{l}\text { Age, smoking, total cholesterol }+ \\
\text { composite definition of IDF } \\
\text { metabolic syndrome (categorical) }\end{array}$ & $2.04(1.47-2.84)$ & $1.62(1.09-2.40)$ & $2.51(1.21-5.22)$ \\
\hline$p$ value & $<0.001$ & 0.017 & 0.013 \\
\hline
\end{tabular}

of the in vivo exposure of an individual, which might be better captured by postprandial triglyceride concentrations. Furthermore, insulin resistance is associated with a wide array of defects beyond dyslipidaemia, hypertension and obesity. Thus novel risk factors, including inflammatory markers such as C-reactive protein [21, 22] or adipokines (e.g. adiponectin and leptin [23]), may be more pertinent to the excess CHD mortality rates in South Asians, but unfortunately were not measured in our study and have also not been examined prospectively in South Asians in any other study. Factors not directly associated with insulin resistance, such as elevated lipoprotein(a), may further account for the South Asian susceptibility to greater CHD [24]. Again, there are no prospective data on South Asians.

Ethnic differences in lifestyle factors such as physical activity could also impact on CHD mortality. These were measured subjectively by self-report in this study, and adjustment for the effect of physical activity had little impact on the elevated CHD risk in South Asians. Notably, physical activity, dietary and other lifestyle factors differ markedly between South Asian subgroups and between South Asian men and women. However, the elevated CHD risk is shared by all South Asian subgroups, indicating that any candidate risk factor must also be shared by these subgroups. However, there are no published studies of the objective measurement of physical activity among South
Asians [25] and this would be a necessary next step to try to ascertain this information more rigorously.

Finally, it also cannot be ruled out that the greater CHD mortality in South Asians is due to poorer healthcare access and treatment, although emerging evidence from the UK points against this likelihood. Thus in 1,350 randomly selected individuals, ethnicity-, sex- or socioeconomicstatus-related factors did not contribute to health-seeking behaviour for chest pain [26]. In the Whitehall-II prospective cohort study of 10,308 British civil servants, the 560 South Asians had higher incident coronary morbidity and mortality than white participants, but Asians were more, not less, likely to have cardiac procedures and to be taking more secondary prevention drugs than their white counterparts, even after adjustment for clinical need [27]. Similar findings were reported in a study from Newcastle, where no evidence was found for an association between social network size and coronary risk factors in Asians or Europeans [28]. In our study, moreover, we attempted to account for the potential effect of ethnic differences in socio-economic status (years of education and occupational social class) on CHD risk.

Importantly, we showed that conventional risk factors, such as smoking, blood pressure and cholesterol, do indeed predict mortality in South Asians as well as in Europeans, underlining the need to attend to these risk factors in any given ethnic group. 


\section{Limitations and strengths}

Our analyses were based on death certificate reporting, with its accompanying limitations. It is known that errors can be made in the recording of the cause of death by the person certifying the death, but we are not aware of any data showing that the rate of such errors varies by ethnic group in the UK. Coding changes between the ICD-10 (introduced 2001) and ICD-9 (1979-2001) revisions are unlikely to have affected how deaths were coded over our study period (1988 to 2006), as, reassuringly, trends in mortality from ischaemic heart disease have been unaffected by the introduction of ICD-10 [29]. As discussed above, the lack in our study of data on the duration of diabetes is a potential limitation, and should be addressed in future studies.

Our study has several strengths. First, ethnic origin was identified by the interviewer on the basis of name, appearance and country of birth, as well as by direct enquiry from participants, and this has an advantage over studies based on routine data, which use country of birth as a proxy for ethnicity. Second, the follow-up rate was an excellent $99.4 \%$. Finally, we were able to examine in detail potential determinants for the excess CHD risk in South Asians, since we had access to a comprehensive battery of baseline risk factors, including surrogate measures of insulin resistance.

\section{Implications}

We suggest that South Asians are markedly sensitive to the impact of diabetes on CHD risk, and therefore prevention of diabetes would have major benefits for this population. In South Asians with diabetes, our data suggest that physicians should be particularly aggressive in the prevention of cardiovascular events. Similarly, in South Asians without diabetes, cardioprotection should begin earlier, or at lower thresholds of risk when assessed by conventional methods, e.g. Framingham-based risk factor charts, as proposed by the recent Joint British Societies guidelines, where cardiovascular risk in South Asians is assumed to be about 1.4 times higher than predicted from the risk charts [30].

As regards the metabolic syndrome, while the concept remains useful in demonstrating elevated pathophysiological risk of $\mathrm{CHD}$, our data suggest that its clinical use in helping to discriminate risk is far from established, as previously argued by ourselves and others [31, 32]. This is even more the case in a context where different definitions of the metabolic syndrome are used [33], different ethnicspecific cut-points are recommended, and their utility in determining the reasons for ethnic differences in CHD risk are unclear.

\section{Conclusion}

These data confirm that CHD mortality in South Asian men is significantly higher than in their European counterparts, but at the same time indicate that conventional risk factors, insulin resistance parameters and metabolic syndrome criteria as currently defined cannot account for this excess risk. Our data reiterate the need to be particularly aggressive in the cardiovascular risk management of South Asians, and to place greater emphasis on the prevention of diabetes in this population. Our findings further highlight the need for studies to determine whether unmeasured factors, associated with insulin resistance or not, are more relevant to the excess CHD mortality rates in South Asians.

Acknowledgements The Southall Study was supported by project grants from the Medical Research Council and the British Heart Foundation. N. G. Forouhi was supported by a Wellcome Training Fellowship in Clinical Epidemiology, the Eastern Deanery Public Health Training Programme, and the Medical Research Council. All analyses were conducted independently of the funding sources.

\section{References}

1. McKeigue PM, Miller GJ, Marmot MG (1989) Coronary heart disease in South Asians overseas: a review. J Clin Epidemiol 42:597-609

2. Wild S, McKeigue P (1997) Mortality by country of birth in England and Wales, 1970-1992. Br Med J 314:689-762

3. McKeigue PM, Ferrie JE, Pierpoint T et al (1993) Association of early-onset coronary heart disease in South Asian men with glucose intolerance and hyperinsulinemia. Circulation 87:152-161

4. McKeigue PM, Shah B, Marmot MG (1991) Relation of central obesity and insulin resistance with high diabetes prevalence and cardiovascular risk in South Asians. Lancet 337:382-386

5. Knight TM, Smith Z, Sahota P et al (1992) Insulin resistance, diabetes, and risk markers for ischaemic heart disease in Asian men and non-Asian men in Bradford. Br Heart J 67:343-350

6. Hughes K, Aw T-C, Kuperan P et al (1997) Central obesity, insulin resistance, syndrome $\mathrm{X}$, lipoprotein(a), and cardiovascular risk in Indians, Malays, and Chinese in Singapore. J Epidemiol Community Health 51:394-399

7. Dhawan J, Bray CL, Warburton R et al (1994) Insulin resistance, high prevalence of diabetes, and cardiovascular risk in immigrant Asians: genetic or environmental effect? Br Heart J 72:413-421

8. Hughes LO, Cruickshank JK, Wright J et al (1989) Disturbances of insulin in British Asian and white men surviving myocardial infarction. Br Med J 299:537-541

9. Chambers JC, McGregor A, Jean-Marie J et al (1999) Abnormalities of vascular endothelial function may contribute to increased coronary heart disease risk in UK Indian Asians. Heart 81: 501-504

10. Hoogeveen RC, Gambhir JK, Gambhir DS et al (2001) Evaluation of $\mathrm{Lp}[\mathrm{a}]$ and other independent risk factors for CHD in Asian Indians and their USA counterparts. J Lipid Res 42:631-638

11. Miller GJ, Beckles GL, Maude GH et al (1989) Ethnicity and other characteristics predictive of coronary heart disease in a 
developing community: principal results of the St James Survey, Trinidad. Int J Epidemiol 18:808-817

12. Collins VR, Dowse GK, Cabealawa S et al (1996) High mortality from cardiovascular disease and analysis of risk factors in Indian and Melanesian Fijians. Int J Epidemiol 25:59-69

13. Chaturvedi N, McKeigue PM, Marmot MG (1993) Resting and ambulatory blood pressure differences in Afro-Caribbeans and Europeans. Hypertension 22:90-96

14. World Health Organization (1999) Definition, diagnosis and classification of diabetes mellitus. Part I. WHO, Geneva

15. International Diabetes Federation (2005) The IDF consensus worldwide definition of the metabolic syndrome. http://www.idf. org/webdata/docs/IDF Metasyndrome definition.pdf (accessed May 2006)

16. Matthews DR, Hosker JP, Rudenski AS et al (1985) Homeostasis model assessment: insulin resistance and beta-cell function from fasting plasma glucose and insulin concentrations in man. Diabetologia 28:412-419

17. UK Prospective Diabetes Study Group (1998) Ethnicity and cardiovascular disease. The incidence of myocardial infarction in white, South Asian and Afro-Caribbean patients with type 2 diabetes (UK Prospective Diabetes Study 32). Diabetes Care 21:1271-1277

18. Chaturvedi N, Fuller JH (1996) Ethnic differences in mortality from cardiovascular disease in the UK: do they persist in people with diabetes? J Epidemiol Community Health 50:137-139

19. Mather HM, Chaturvedi N, Fuller JH (1998) Mortality and morbidity from diabetes in South Asians and Europeans: 11-year follow-up of the Southall Diabetes Survey, London, UK. Diabet Med 15:53-59

20. Mukhopadhyay B, Forouhi NG, Fisher BM et al (2006) Glycaemic control over time among European and South Asian patients with type 2 diabetes: results from follow up in a routine diabetes clinic. Diabetic Med 23:94-98

21. Chambers JC, Eda S, Bassett P et al (2001) C-reactive protein, insulin resistance, central obesity, and coronary heart disease risk in Indian Asians from the United Kingdom compared with European whites. Circulation 104:145-150
22. Forouhi NG, Sattar N, McKeigue PM (2001) Relation of Creactive protein to body fat distribution and features of the metabolic syndrome in Europeans and South Asians. Int J Obes Relat Metab Disord 25:1327-1331

23. Abate N, Chandalia M, Snell PG et al (2004) Adipose tissue metabolites and insulin resistance in nondiabetic Asian Indian men. J Clin Endocrinol Metab 89:2750-2755

24. Bhatnagar D, Anand IS, Durrington PN et al (1995) Coronary risk factors in people from the Indian subcontinent living in West London and their siblings in India. Lancet 345:405-409

25. Fischbacher CM, Hunt S, Alexander L (2004) How physically active are South Asians in the United Kingdom? A literature review. J Public Health (Oxf) 26:250-258

26. Adamson J, Ben Shlomo Y, Chaturvedi N et al (2003) Ethnicity, socio-economic position and gender-do they affect reported health-care seeking behaviour? Soc Sci Med 57:895-904

27. Britton A, Shipley M, Marmot M et al (2004) Does access to cardiac investigation and treatment contribute to social and ethnic differences in coronary heart disease? Whitehall II Prospective Cohort Study. Br Med J 329:318

28. Pollard TM, Carlin LE, Bhopal R et al (2003) Social networks and coronary heart disease risk factors in South Asians and Europeans in the UK. Ethn Health 8:263-275

29. Office for National Statistics (2004) The impact of introducing ICD-10 on trends in mortality from circulatory diseases in England and Wales. Health Stat Q 22:14-20

30. Joint British Societies (2005) JBS 2: Joint British Societies' guidelines on prevention of cardiovascular disease in clinical practice. Heart 91 (Suppl 5):v1-v52

31. Sattar N, Forouhi NG (2005) Metabolic syndrome criteria: ready for clinical prime time or work in progress? Eur Heart J 26:1249-1251

32. Greenland P (2005) Critical questions about the metabolic syndrome. Circulation 112:3675-3676

33. Lawlor DA, Smith GD, Ebrahim S (2006) Does the new International Diabetes Federation definition of the metabolic syndrome predict CHD any more strongly than older definitions? Findings from the British Women's Heart and Health Study. Diabetologia 49:41-48 